\title{
USOS DE PLANTAS MEDICINALES EN LA COMUNIDAD SAN JACINTO DEL CANTÓN VENTANAS, LOS RIOS - ECUADOR
}

\section{USE OF MEDICINAL PLANTS IN THE SAN JACINTO COMMUNITY - VENTANAS MUNICIPALITY, LOS RÍOS - ECUADOR}

\author{
Daniel Jacob Paredes ${ }^{1}$, Mónica Patricia Buenaño-Allauca², Néstor Javier Mancera-Rodríguez ${ }^{3}$
}

\begin{abstract}
${ }^{1}$ Ingeniero en Ecoturismo, Universidad Técnica Estatal de Quevedo UTEQ, Ecuador, e-mail: danjacob24@hotmail.com;
${ }^{2}$ Magister en Planificación y Gestión de Proyectos Agroturísticos y Ecológicos, Profesor Ingeniería en Ecoturismo, Universidad Técnica Estatal de Quevedo UTEQ, Ecuador, e-mail: monicauteq@gmail.com; ${ }^{3}$ Doctor en Biología, Profesor Titular. Universidad Nacional de Colombia, Departamento de Ciencias Forestales, Sede Medellín, Calle 59A No. 63-20, Bloque 20, oficina 211, Medellín, Colombia, Investigador Prometeo, e-mail: njmancer@unal.edu.co
\end{abstract}

Rev. U.D.C.A Act. \& Div. Cient. 18(1): 39-50, Enero-Junio, 2015

\section{RESUMEN}

Se investigó el uso de plantas medicinales en la Comunidad San Jacinto, provincia de Los Ríos (Ecuador). Se estableció qué partes de las plantas son usadas, el tipo de administración, la preparación, las categorías de uso medicinal tradicional y el conocimiento por sexo y por rangos de edad. Se determinó el valor de uso (IVU), el conocimiento relativo (RVU) y el nivel de uso significativo Tramil (UST). Se registran 33 plantas que tuvieron uso medicinal; las hojas fueron las estructuras más utilizadas (87,9\%); la forma de preparación principal fue la infusión $(66,7 \%$ ) y la vía de administración más empleada fue la bebida (87,9\%). La categoría de uso medicinal mejor representada está relacionada con el sistema gastrointestinal $(36,4 \%)$. No se encontró diferencias significativas en el conocimiento de las especies de acuerdo al sexo y al rango de edad de los informantes. Las plantas medicinales más importantes para la población local fueron: la sábila (Aloe vera), la hoja del aire (Kalanchoe pinnata), la ruda de gallinazo (Ruta graveolens), el toronjil (Melissa officinalis), la valeriana (Valeriana officinalis), la hierba luisa (Cymbopogon citratus) y el orégano (Origanum vulgare).

Palabras clave: Medicina tradicional, etnobotánica, análisis cuantitativo.

\section{SUMMARY}

The use of medicinal plants by San Jacinto community, in Los Rios Province (Ecuador), was investigated. The importance of plant parts, the type of administration, the prepa- ration, the categories of traditional medicinal use and the species knowledge by sex and age, were established. Use value of species (IVU), the relative knowledge by several informants (VUR) and the level of meaningful use Tramil (UST) were determinate. Thirty three medicinal plants were recorded, the most commonly used structures used in the preparation of medicine were the leaves (87.9\%), the major form to preparation was the infusion $(66.7 \%)$, and the route of administration was drink (87.9\%). The medicinal usage category best represented is related with the gastrointestinal system (36.4\%). No significant difference in knowledge of species according to sex and age range of the respondents was found. The most important medicinal plants for local people were Aloe vera, Kalanchoe pinnata, Ruta graveolens, Melissa officinalis, Valeriana officinalis, Cymbopogon citratus and Origanum vulgare.

Key words: Traditional medicine, ethnobotanical, quantitative analysis.

\section{INTRODUCCIÓN}

Las plantas medicinales tienen una contribución importante en el sistema de salud de comunidades locales, ya que son usadas, de manera frecuente, por la mayoría de las poblaciones rurales (e.g., Moncayo et al. 2006; Angulo et al. 2012). En la actualidad, un gran porcentaje de la población mundial, en particular en países en desarrollo, emplean plantas para afrontar las necesidades primarias de asistencia médica (Tene et al. 2007). Asimismo, las plantas han jugado un papel fundamental en el desarrollo de las culturas andinas, siendo utilizadas como fuente de alimento, de 
medicinas, de combustible, de materiales de construcción, de herramientas e, incluso, un lugar importante en su sistema de creencias y de ritos (Almeida, 2000; Cerón Martínez, 2006; de la Torre et al. 2006; 2008).

Ecuador es considerado uno de los países con mayor biodiversidad en el mundo (Jorgensen \& León-Yánez, 1999), lo que le confiere un gran potencial en el uso de las plantas, como fuente de medicina tradicional, siendo importante establecer aspectos, como las formas de uso y los beneficios curativos que brindan, en las diferentes comunidades del país. En este sentido, la etnobotánica es una herramienta útil para el rescate del conocimiento sobre el uso del recurso vegetal y de las interacciones que se establecen entre el hombre y las plantas, a través del tiempo (Lagos-López, 2007), adquiriendo interés e importancia su estudio en bosques tropicales, en las últimas décadas, debido a la pérdida acelerada e irreparable del conocimiento tradicional y a la degradación de los bosques (Phillips \& Gentry, 1993).

En Ecuador, los estudios de plantas medicinales y etnobotánica han sido desarrollados, principalmente, en la región central andina o de sierra (Naranjo \& Escaleras, 1995; Naranjo \& Crespo, 1997; Béjar et al. 2002; Bussmann \& Sharon, 2006; Cerón-Martínez, 2006; de la Torre et al. 2006; 2008; Ríos et al. 2008; Armijos et al. 2014.) y en la región de la Amazonía (Villacrés, 1995; Cerón \& Montalvo, 1998; Santín, 2004) y consisten, mayormente, en la enumeración de las plantas que conoce y usa la gente local o la descripción de los sistemas de salud tradicional, en comunidades indígenas. Para la región occidental o costa son pocos los estudios etnobotánicos realizados en comunidades campesinas no indígenas, que han establecido interrelación con su ambiente natural usando plantas medicinales, dentro de sus culturas (Estrella, 1991; Madsen et al. 2001).

El conocimiento campesino sobre las especies nativas y sus usos en la medicina tradicional en áreas rurales ha ido disminuyendo, progresivamente, de manera conjunta con el proceso de pérdida y de degradación de los ecosistemas naturales (CESA, 1992; Cerón-Martínez, 2002) y, dichos conocimientos, no se trasmiten, adecuadamente, a las nuevas generaciones. Rivera \& Obón (1991) destacan, de igual manera, que el desarrollo humano está haciendo que se pierda la cultura tradicional de utilizar las plantas curativas, como lo hacían los antepasados. Por lo anterior, es importante conocer y rescatar el conocimiento del uso de las plantas en la medicina tradicional, siendo el objetivo del presente estudio, establecer el estado actual del conocimiento local sobre el uso de plantas medicinales en las comunidades campesinas del área rural de la Comunidad San Jacinto del cantón Ventanas, provincia de Los Ríos, Ecuador. La importancia del desarrollo de este tipo de estudios en comunidades no indígenas ha sido destacada en diversos trabajos (Pinedo-Vásquez et al. 1990; Phillips \& Gentry, 1993; Bellon, 1996; Galeano, 2000; Marín-Corba et al. 2005; Hurtado-(lloa \& Moraes, 2010), puesto que la mayoría de estudios, a nivel mundial, han sido desarrollados en grupos indígenas.

\section{MATERIALES Y MÉTODOS}

La investigación, se llevó a cabo en la Comunidad de San Jacinto del Cantón Ventanas, en la provincia de Los Ríos, en la región litoral del Ecuador, a una distancia de $11 \mathrm{~km}$ del cantón Ventanas, ubicado a $01^{\circ} 24^{\prime} 54,80^{\prime \prime}$ de latitud Sur y $79^{\circ} 21^{\prime} 24,89^{\prime \prime}$ longitud Oeste, altitud de $217 \mathrm{msnm}$ (Figura 1). Se ubica en una zona de vida de bosque húmedoTropical (Holdridge, 1987). El período seco, se presenta de julio a diciembre y el período de invierno, de enero a junio, y la temperatura varía entre los 17 y $34^{\circ} \mathrm{C}$. Según el Censo de Población y Vivienda del 2010, el Cantón Ventanas tenía 28.383 habitantes en el área rural (INEC, 2010), dedicados, principalmente, a actividades del sector agropecuario $(42,1 \%)$, como el cultivo de maíz, de banano, de soya y de cacao.

La Comunidad de San Jacinto es naturaleza étnica autodefinida como Montubia; tiene una población mestiza cercana a los 600 habitantes, dispersos y distanciados unos de otros en el área rural y hace parte de la parroquia de Los Ángeles, conformada por una población rural de 2.151 habitantes. La zona ha sido altamente modificada por la actividad agropecuaria, presentando pocos remanentes de bosque secundario, altamente intervenido. Producen maíz, soya, banano, cacao, plátano, yuca y se dedican a la pesca y cría de especies menores, para el autoconsumo.

Entre marzo y mayo del 2014, se recolectó información sobre las plantas medicinales cultivadas en huertos y el uso dado por miembros de la comunidad, aplicando encuestas semiestructuradas, con entrevistas a personas identificadas por los líderes de la comunidad, como poseedoras de un alto conocimiento en la medicina tradicional y con cultivo en huertos de plantas medicinales. Las encuestas semiestructuradas permiten obtener información directa del entrevistado, mediante una serie de preguntas cerradas, que ayudan a orientar las conversaciones y, otras abiertas, que permiten indagar por aspectos complementarios.

Se indagó sobre las plantas empleadas para tratar enfermedades, los usos medicinales tradicionales, los métodos de preparación, la vía de administración y las partes de la planta. Además, se obtuvo información sobre aspectos socioculturales, tales como la edad y el género. Se realizó una prueba de Chi-cuadrado $\left(\mathrm{x}^{2}\right)$, mediante el software PAST (Hammer y Harper, Oslo, Noruega) (Hammer et al. 2001), para determinar las diferencias significativas en la impor- 


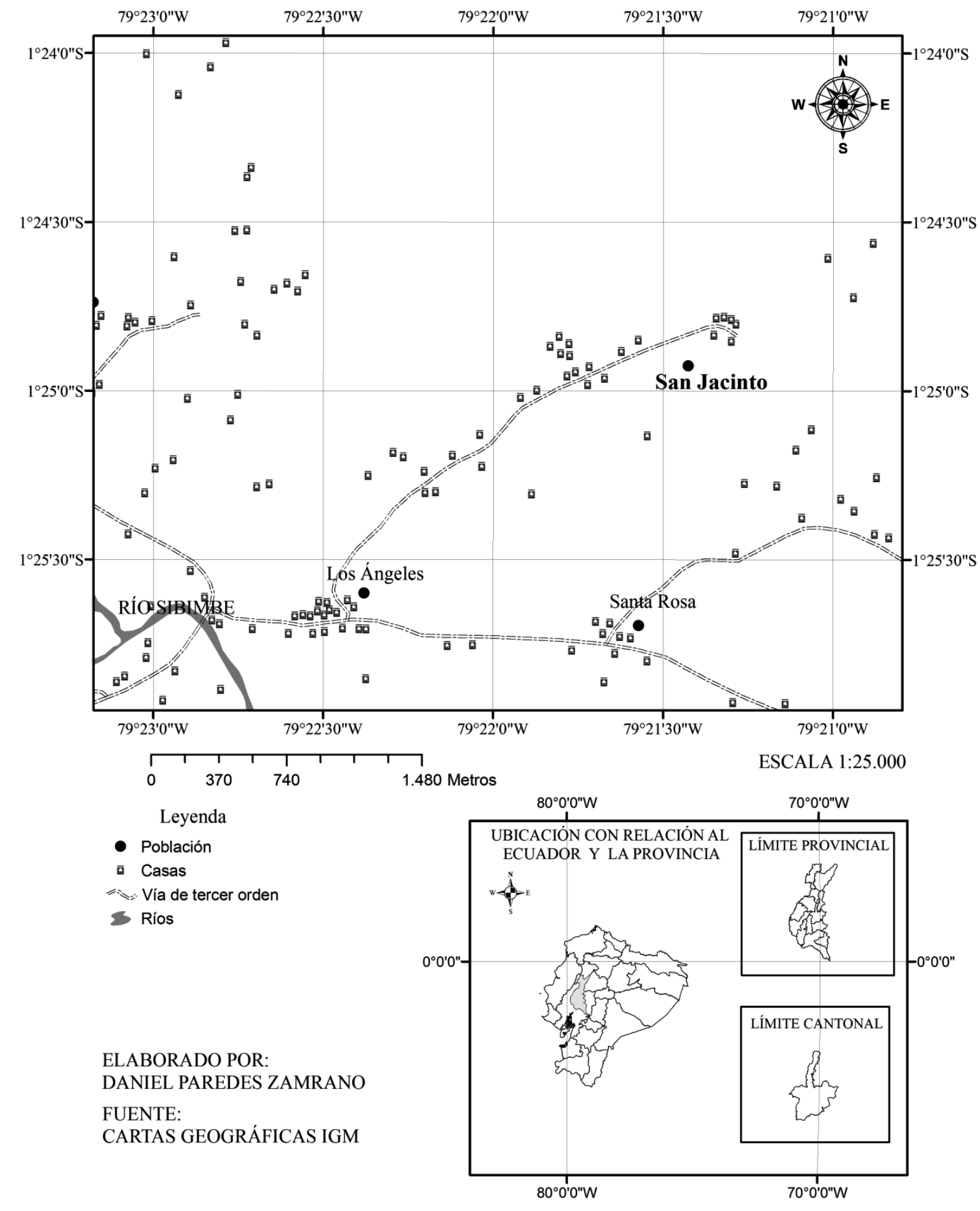

Figura 1. Localización de la Comunidad San Jacinto del cantón Ventanas, provincia de Los Ríos, Ecuador. 
tancia de las partes de las plantas usadas por la comunidad (cristal, flor, fruto, hojas, raíz, semilla, tallo), el método de preparación (cocción: se hierve la planta en agua por varios minutos, puede ser de uso local o por vía oral; emplasto: aplicación tópica directa de la planta medicinal fresca o cocinada, triturada o machacada; infusión: al hervir el agua, se adicionan las plantas medicinales, se deja en reposo por 10 a 15 minutos, puede ser de uso local o por vía oral; jugo: líquido que se exprime luego de machacar o triturar una planta fresca, puede ser de uso local o por vía oral; macerado: remojo por varios días de la planta medicinal triturada, en solventes como agua, aceite o alcohol); la vía de administración (aromaterapia, bebida, comida, uso externo) y el conocimiento de las especies por rangos de edad (rango I: entre 19 y 54 años, $n=23$; rango II: entre 55 y 77 años, $n$ $=23$ ) y género de las personas entrevistadas.

Se realizó caminatas en los huertos con cada informante, donde se reconocieron y se colectaron las plantas medicinales. A cada una de ellas, se le hizo la correspondiente descripción botánica, para su posterior determinación taxonómica, registrando el nombre común, el lugar de colecta, la enfermedad o afección que remedia y el proceso de uso. Se llevó a cabo el proceso de herborización (colecta, secado y montaje), en el Herbario de la Universidad Técnica Estatal de Quevedo (UTEQ). Las muestras secas fueron identificadas, considerando el sistema de clasificación APG (Angiosperm Phylogeny Group) (Stevens, 2001-2013), utilizando literatura especializada (Gentry, 1982) y consulta a expertos.

Las enfermedades o afecciones tratadas por la etnomedicina, mencionadas por los habitantes, se clasificaron de acuerdo a las categorías propuestas por Bhattarai et al. (2010) y Angulo et al. (2012), en las siguientes once categorías, relacionadas al sistema del cuerpo, donde las plantas medicinales controlan la afección: ENT (Sistema sensorial), Gastrointestinal, Hepático, Inmunológico, Neurológico, Osteomuscular, Otros sistemas, Piel, Renal/urológico, Reproductivo y Respiratorio (Tabla 1).

Tabla 1. Porcentaje de uso medicinal y enfermedades tratadas con plantas medicinales, agrupadas por sistema del cuerpo, por los habitantes del área rural de la Comunidad San Jacinto, provincia de Los Ríos, Ecuador.

\begin{tabular}{|c|c|c|}
\hline $\begin{array}{l}\text { Sistema del cuerpo } \\
\text { (categorías) }\end{array}$ & $\begin{array}{l}\text { Enfermedades o afecciones tratadas por la etnomedicina según } \\
\text { la información de los habitantes }\end{array}$ & $\%$ de uso \\
\hline ENT (Sistema sensorial) & $\begin{array}{l}\text { Dolores en nariz, ojos y oídos, sinusitis y dolor dental. Problemas de concen- } \\
\text { tración, cansancio excesivo, problemas de sueño, preocupación y tristeza. }\end{array}$ & $9,1 \%$ \\
\hline Gastrointestinal & Diarrea, vómitos, dolor de estómago, gastritis, parásitos. & $36,4 \%$ \\
\hline Hepático & Enfermedades hepáticas, hígado graso. & $6,1 \%$ \\
\hline Inmunológico & Enfermedades autoinmunes, cáncer, enfermedades infecciosas. & $3,0 \%$ \\
\hline Neurológico & Adormecimiento de las extremidades, parálisis, enfermedad de Parkinson. & $3,0 \%$ \\
\hline Osteomuscular & $\begin{array}{l}\text { Fracturas óseas, dolor de huesos, enfermedades óseas, dolor en las articu- } \\
\text { laciones y cintura, inflamación del cuerpo, reumatismo, dolor de cuello y ex- } \\
\text { tremidades. }\end{array}$ & $21,2 \%$ \\
\hline Otros & $\begin{array}{l}\text { Fiebre (cualquier tipo de fiebre), dolor de cabeza, vértigo/mareos, dejar de } \\
\text { sudar, la diabetes, mordedura de serpiente, "dolor de hinchazón", caída del } \\
\text { cabello, desinflamación y para bajar de peso. }\end{array}$ & $18,2 \%$ \\
\hline Piel & Infecciones, heridas e inflamaciones en la piel. & $12,1 \%$ \\
\hline Renal/urológico & $\begin{array}{l}\text { Enfermedades renales, infección del tracto urinario, diurético, desinflamación } \\
\text { de la próstata. }\end{array}$ & $12,1 \%$ \\
\hline Reproductivo & $\begin{array}{l}\text { Infertilidad, heridas en la vagina, trabajo de parto, detener el sangrado du- } \\
\text { rante el parto, trastornos de la menstruación (flujo abundante), problemas } \\
\text { menstruales. }\end{array}$ & $3,0 \%$ \\
\hline Respiratorio & $\begin{array}{l}\text { Dificultad para respirar, dolor de garganta, dolor de pecho, gripe, bronquitis, } \\
\text { tos y resfríos. }\end{array}$ & $21,2 \%$ \\
\hline
\end{tabular}


La información etnobotánica proporcionada por los habitantes del área de estudio fue organizada en una base de datos, empleando una hoja de cálculo de Microsoft Office Excel 2007. Los porcentajes y las frecuencias de las citaciones de las plantas medicinales asociadas al conocimiento tradicional, fueron utilizados en el cálculo de los tres índices de uso más común en estudios etnobotánicos, para determinar la importancia de las especies. El cálculo de los porcentajes de cada categoría de uso, método de preparación, vía de administración y parte de la planta, se realizó teniendo en cuenta, para cada uno de ellos, el número de citaciones en cada planta con relación al total de citaciones para todas las plantas.

Índice de valor de uso de especies. Expresa la importancia o valor cultural de una especie para todos los informantes entrevistados (Phillips \& Gentry, 1993; Phillips, 1996). Para estimar el índice de valor de uso general de cada especie para todos los informantes (IVUs), se utilizó la fórmula

$$
I V U s=\frac{\sum i V U i s}{N s}
$$

Dónde: $V U i s=$ valor de uso de la especie por cada informante, y $N_{s}=$ es el número de informantes para cada especie.

Conocimiento relativo de la especie por varios informantes (RVU). Se utilizó la siguiente formula, representada por Philips (1996).

$$
R V U=\frac{\Sigma \frac{V U i s}{I V U s}}{N s p}
$$

Dónde: $V U i s=$ valor de uso de la especie por cada informante, $I V U s=$ es el índice de valor de uso de la especie y $\Lambda s p=$ es el número de especies.

Nivel de uso significativo Tramil (UST). Para estimar el nivel de uso para cada especie y verificar su aceptación cultural, se utilizó la metodología propuesta por GermosénRobineau (1995). Ésta expresa que aquellos usos medicinales que son citados con una frecuencia superior o igual al $20 \%$, por las personas encuestadas que usan plantas como primer recurso para un determinado problema de salud, se pueden considerar significativos, desde el punto de vista de su aceptación cultural y, por lo tanto, merecen su evaluación y validación científica (Toscano-González, 2006). El UST, se calcula mediante la siguiente ecuación:

$$
U S T=\frac{U \text { so especie }(s)}{\text { Nis }} \times 100
$$

Dónde: Uso especie (s) = número de citaciones para cada especie y Nis $=$ número de informantes encuestados.

\section{RESULTADOS Y DISCUSIÓN}

La información sobre las plantas medicinales y el uso dado por miembros de la comunidad, se obtuvo mediante una muestra representada por 25 hombres y 21 mujeres, quienes tuvieron conocimientos sobre plantas medicinales, con un rango de edad de los informantes hombres, de 26 a 77 años y, de mujeres, de 19 a 74 años. A partir de la información, se obtuvo el registro de un total de 33 especies de plantas medicinales, identificadas hasta nivel de especie, distribuidas en 21 familias y 31 géneros (Cuadro 1). Las familias con mayor número de especies medicinales, en todo el estudio, fueron Lamiaceae (seis especies - 18,2\%), seguida por Rutaceae (tres especies - 9,1\%), las familias Amaranthaceae, Apiaceae, Crassulaceae, Fabaceae, Rubiaceae y Verbenaceae estuvieron representadas con dos especies cada una, constituyendo estas 6 familias, en conjunto, el $36,42 \%$, del total de las especies, lo que permite inferir la importancia de estas familias, desde el punto de vista etnomedicinal.

Esto coincide con lo reportado por Toscano-González (2006), para el departamento de Boyacá, Colombia, quien reporta dentro de las familias más representativas a Lamiaceae, Rutaceae y Apiaceae. Por su parte, Bernal et al. (2011) relacionan las familias Asteraceae, Fabaceae, Rubiaceae, Solanaceae, y Lamiaceae como las que tienen un mayor número de especies de plantas medicinales en Colombia y para Ecuador, las cinco familias con mayor número de especies de plantas medicinales, son Asteraceae, Fabaceae, Rubiaceae, Solanaceae y Araceae (de la Torre et al. 2008).

Las especies de la familia Lamiaceae son las más utilizadas y su importancia puede estar relacionada tanto con su fácil disponibilidad y a que son las más utilizadas en medicina tradicional, debido a las glándulas con aceites de terpenos, que se encuentran en sus células epidérmicas, con propiedades organolépticas y actividades, tanto antioxidantes como antimicrobianas (Domínguez-Vásquez \& Castro-Ramírez, 2002; Saldanha et al. 2005). Otros estudios, también destacan el interés etnomedicinal de la familia Lamiaceae (Moncayo et al. 2006; Angulo et al. 2012).

Con base en las encuestas realizadas en el área rural de la Comunidad San Jacinto, se evidenció que las hojas son las estructuras más empleadas en la preparación de las medicinas, para contrarrestar diferentes enfermedades, con un 


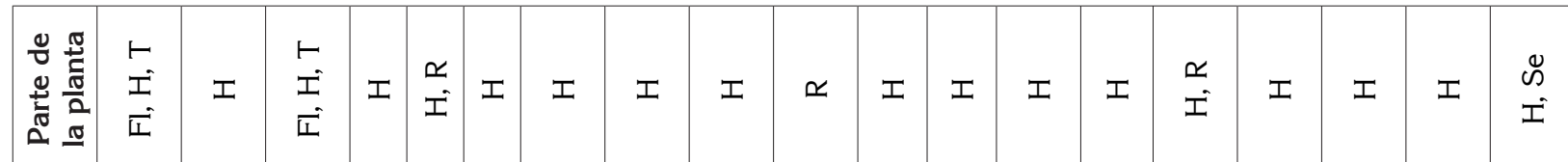
.

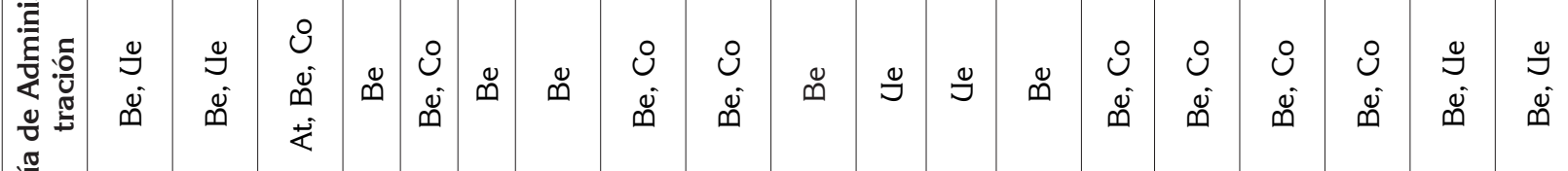
i $>$ $i^{2}$

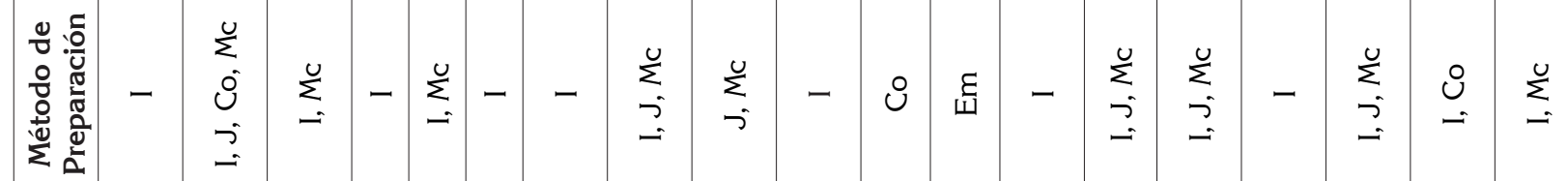

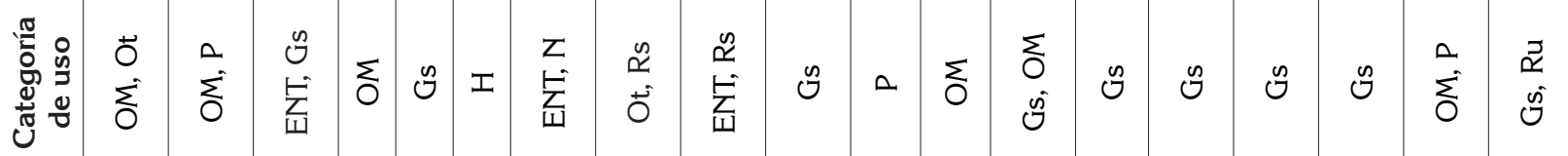

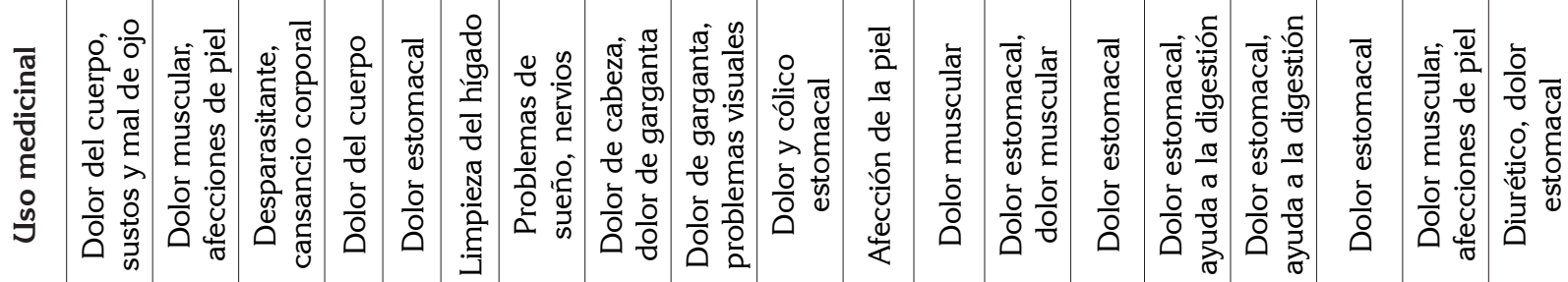
줄

$\frac{0}{\sqrt[0]{0}}$

0
0
0
0
0
0
0
0
0
0
0
0
0
0
0
0
0
0
0
0

\begin{tabular}{|c|c|c|c|c|c|c|c|c|c|c|c|c|c|c|c|c|c|c|c|}
\hline $\begin{array}{l}\frac{\pi}{\mathbb{*}} \\
\frac{\pi}{\alpha}\end{array}$ & 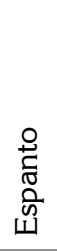 & 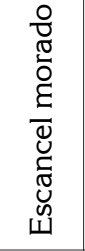 & 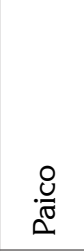 & 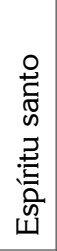 & $\begin{array}{l}\frac{0}{2} \\
\frac{0}{2} \\
\frac{\pi}{3} \\
\text { U. }\end{array}$ & $\begin{array}{l}\stackrel{0}{\vec{T}} \\
\stackrel{0}{\vec{\alpha}}\end{array}$ & 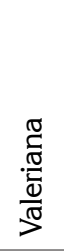 & 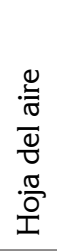 & 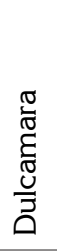 & $\begin{array}{l}\mathbb{N} \\
\text { S } \\
\mathbb{D} \\
N\end{array}$ & 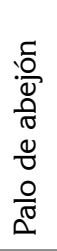 & $\begin{array}{l}0 \\
\frac{0}{0} \\
\frac{0}{0} \\
\text { D̃ } \\
\tilde{D} \\
\text { D }\end{array}$ & $\begin{array}{l}: \overline{\widetilde{\Xi}} \\
0 \\
\stackrel{0}{0}\end{array}$ & $\begin{array}{l}\stackrel{\pi}{\mathbb{E}} \\
\sum\end{array}$ & 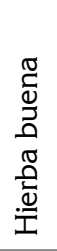 & 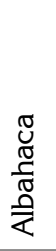 & 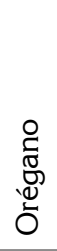 & 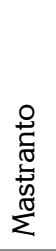 & 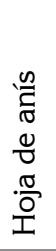 \\
\hline 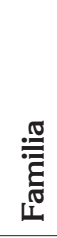 & 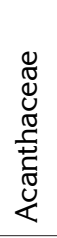 & 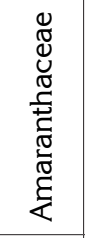 & 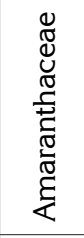 & 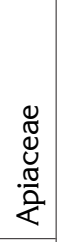 & 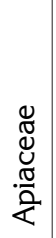 & 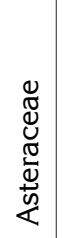 & 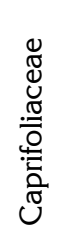 & 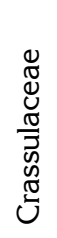 & 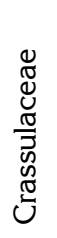 & $\begin{array}{l}\mathbb{\Xi} \\
\mathbb{\Xi} \\
\tilde{\mathbb{E}} \\
\stackrel{0}{\mathbb{E}}\end{array}$ & 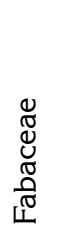 & 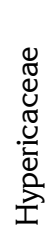 & 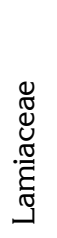 & 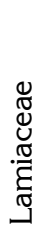 & 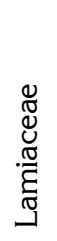 & 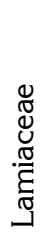 & 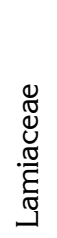 & 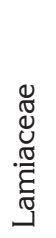 & 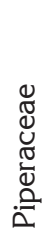 \\
\hline 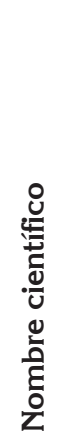 & 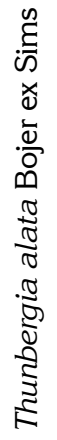 & 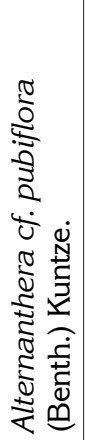 & 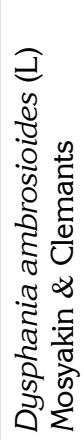 & 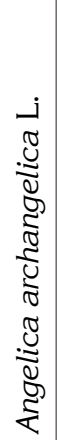 & 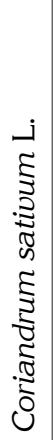 & 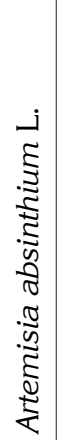 & 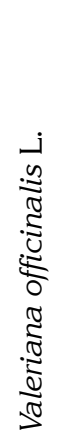 & 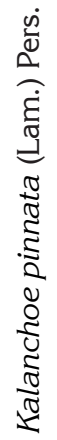 & 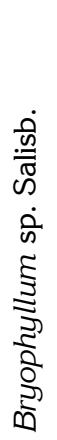 & 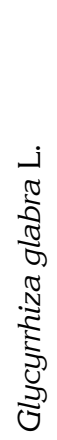 & 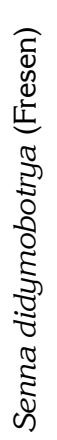 & 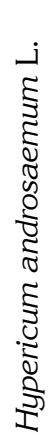 & 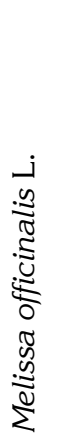 & 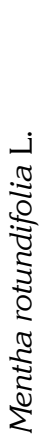 & 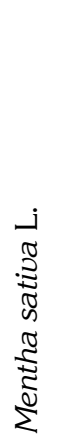 & 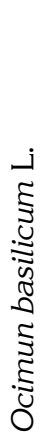 & 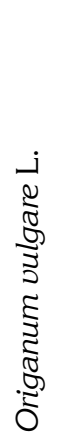 & 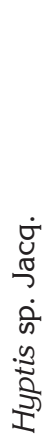 & 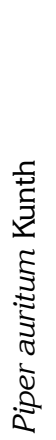 \\
\hline
\end{tabular}




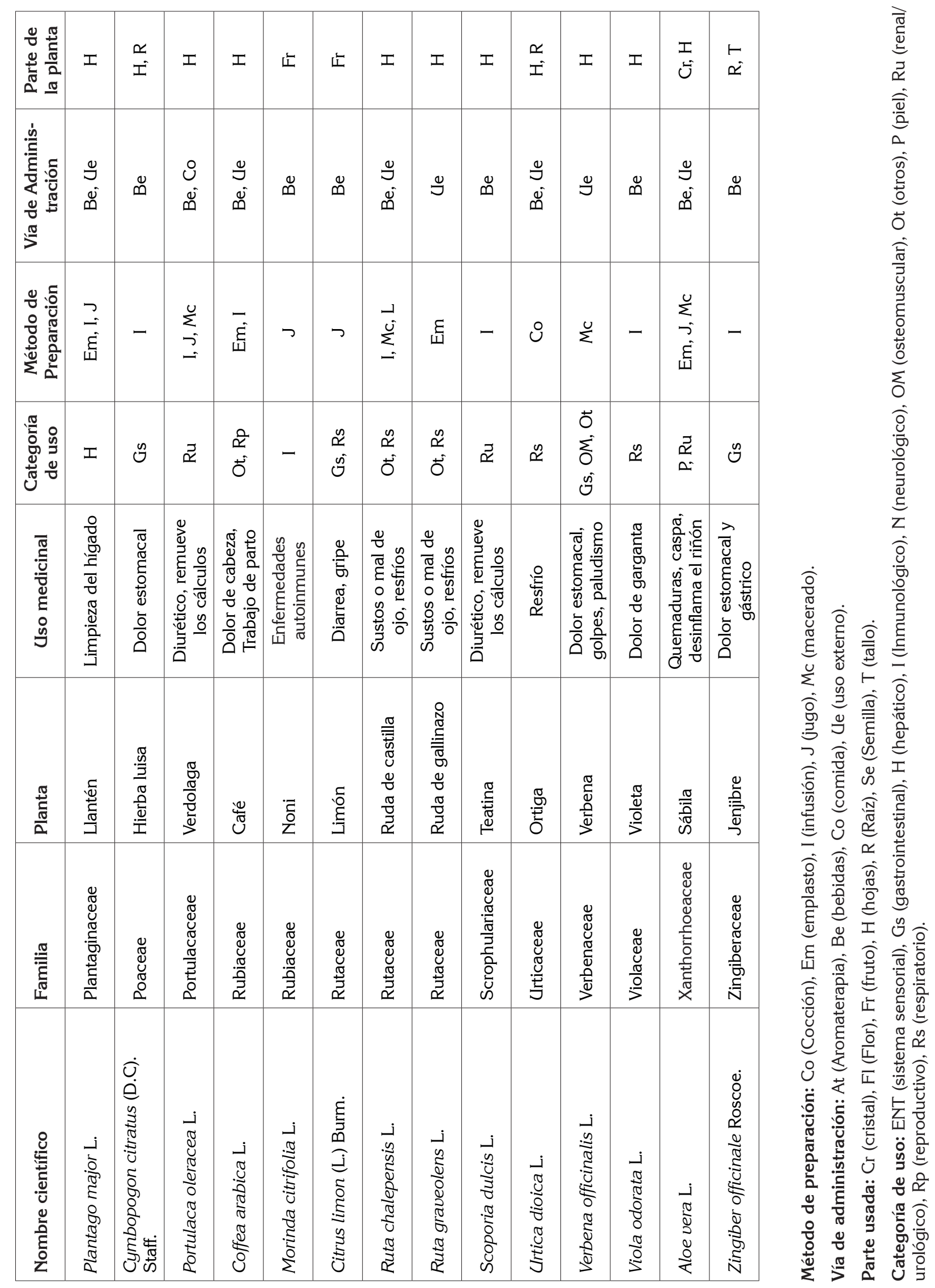


$87,9 \%$ del total de menciones, seguido por la utilización de la raíz $(18,2 \%)$, el tallo $(9,1 \%)$, flores $(6,1 \%)$, frutos $(6,1 \%)$, cristales y semillas (3,0\%, cada uno). La desviación estándar $\left(\mathrm{S}^{2}\right)$ fue de 30,8 y el coeficiente de variación (Cv) de 1,61. La prueba de $\chi^{2}$ muestra que existen diferencias significativas en la importancia de las partes usadas por la comunidad $\left(X^{2}\right.$ cal $=409,27 ; X^{2}$ th $\left.=12,59 ; \mathrm{gl}=6, \mathrm{p}<0,001\right)$. Esto coincide con lo encontrado en otros estudios, como los de Akarreta et al. (2007), Lulekal et al. (2008), de la Torre et al. 2008 y Angulo et al. (2012). Esto se puede deber, principalmente, a que estos órganos almacenan eficientemente una mayor cantidad de compuestos químicos en forma de metabolitos secundarios, con actividad biológica variada (Bhattarai et al. 2010). Angulo et al. (2012), en áreas del sur de Colombia, donde también hay un predominio de especies cultivadas en huertos, presenta coincidencias en la composición de algunas de las especies de este estudio, lo que puede contribuir con estas semejanzas.

Con relación a la clasificación de formas de crecimiento, se evidenció que del total de plantas medicinales mencionadas $(\mathrm{n}=33)$, las hierbas están representadas por 28 especies $(84,8 \%)$, mientras que los arbustos, por tres especies $(9,1 \%)$ (café, paico y palo de abejón) y los árboles, por dos especies $(6,1 \%)$ (limón y noni), coincidiendo con lo reportado por de la Torre et al. (2008) y por Angulo et al. (2012).

Los métodos de preparación para el uso de las plantas medicinales son muy variados. La mayoría son realizadas como infusión, con un 66,7\%; macerado, con 39,43\%; seguida de jugos, con un porcentaje de 33,3\%; en emplasto y cocción, con 15,2\%, cada uno). La desviación estándar $\left(\mathrm{S}^{2}\right)$ fue de 20,3 y el coeficiente de variación (Cv) de 0,77. La prueba de $\chi^{2}$ muestra que existen diferencias significativas en las formas de preparación de las plantas medicinales por la comunidad $\left(X^{2} \mathrm{cal}=261,7 ; \mathrm{gl}=4, \mathrm{p}<0,001\right)$. Esto coincide con lo reportado por Angulo et al. (2012), en el sur de Colombia; sin embargo, difiere de lo encontrado por Arango (2004), en Salento (Quindío), por Moncayo et al. (2006), en el municipio de Chachagüí (Nariño) y por Beyra et al. (2002), en Camagüey (Cuba), quienes reportan, como principal forma de preparación, la cocción y, en menor medida, otras formas, como la infusión, el macerado y el jugo.

En relación con la información recopilada, las personas de la comunidad utilizan siempre plantas frescas en el momento de la preparación y no mencionan el uso de material vegetal seco. A diferencia de lo encontrado en otros estudios, como los de Beyra et al. (2002) o Hernández \& Volpato (2004), no se registró la preparación mediante la mezcla de varias especies de plantas ni el uso de otros excipientes, como aceite vegetal comestible, miel de abeja, clara y yema de huevo, resina de árboles o arbustos, sal, azúcar, grasa de carnero o de vaca, leche de vaca, entre otros.
La vía de administración más empleada es la bebida, con un $87,9 \%$; seguida del uso externo, con $39,4 \%$; de la comida, con $27,3 \%$ y la aromaterapia, con 3,0\%. La desviación estándar $\left(S^{2}\right)$ fue de 32,8 y el coeficiente de variación $(C v)$ de 1,02. La prueba de $x^{2}$ muestra que existen diferencias significativas en las vías de administración de las plantas medicinales por la comunidad $\left(X^{2} \mathrm{cal}=266,2\right.$; $\mathrm{gl}=3$; $\mathrm{p}<$ $0,001)$. La vía de administración muestra que la vía oral (bebida y comida) es la más usada por los habitantes de la zona, porque facilita una asimilación más rápida de las propiedades medicinales de las plantas usadas.

De las once categorías del sistema del cuerpo humano que presentan mayor número de especies de plantas medicinales empleadas para el tratamiento de enfermedades o afecciones relacionadas con éstas son: el sistema gastrointestinal, con $36,4 \%$ y el sistema osteomuscular y el sistema respiratorio, con $21,2 \%$, cada uno (Tabla 1). La desviación estándar $\left(\mathrm{S}^{2}\right)$ fue de 10,34 y el coeficiente de variación $(\mathrm{Cv})$ de 0,78 . La prueba de $x^{2}$ muestra que existen diferencias significativas entre las categorías de uso medicinal por la comunidad $\left(X^{2}\right.$ cal $=138,38 ; X^{2}$ th $=18,31 ; g l=10, p<$ 0,001).

En la comunidad de San Jacinto existe un valioso conocimiento tradicional sobre plantas medicinales, que permiten su uso en el tratamiento de diversos problemas de salud, siendo las afecciones o las enfermedades digestivas, parasitosis intestinal y enfermedades respiratorias, las más tratadas con éstas. Igualmente, se atienden otra gran variedad de afecciones de los sistemas, renal/urológico, piel, osteomuscular, entre otros. Esto es similar a lo hallado en otros estudios, como el realizado por Valdés-Cobos (2013), en México, donde las principales enfermedades tratadas con plantas medicinales son las relacionadas con los problemas de salud de índole gastrointestinal y respiratorio y, el de Angulo et al. (2012), en Colombia, en el que las enfermedades más atendidas con plantas medicinales fueron las gastrointestinales. Es de destacar que, en estos casos, las poblaciones humanas son pertenecientes a comunidades rurales de escasos recursos económicos, con problemas de desnutrición infantil, carencia de servicios médicos de calidad y sin acceso a servicios básicos de primera necesidad, como agua potable y servicios sanitarios adecuados, lo que hace que enfermedades respiratorias y gastrointestinales, que podrían ser fácilmente tratadas mediante programas de salud pública, sean comunes, debido a problemas de insalubridad.

En relación con la información recopilada de acuerdo al género de las personas de la comunidad encuestadas, se registró un total de 30 especies mencionadas por las mujeres $(90,9 \%)$ y 25 especies por los hombres $(75,8 \%)$. La prueba de $\mathrm{x}^{2}$ muestra que no existen diferencias significa- 
tivas en el conocimiento de hombre y mujeres en el número de plantas de uso medicinales conocidas $\left(X^{2} \mathrm{cal}=2,21\right.$; $X^{2}$ th $\left.=3,84 ; \mathrm{gl}=1 ; \mathrm{p}=0,137\right)$. Esto difiere de lo reportado por Arango (2004), en Salento (Quindío), en la vertiente occidental de la Cordillera Central de los Andes colombianos, por Suárez-Duque (2008), en la comunidad de San Jacinto de Chinambí, en la provincia del Carchi, Ecuador y por Angulo et al. (2012), en el corregimiento de Genoy, Municipio de Pasto, Colombia, quienes encontraron que las mujeres tienen un mayor conocimiento de plantas de uso medicinal. Otros estudios han registrado que los hombres son los que tienen más conocimiento sobre usos de especies del bosque, principalmente árboles, mientras las mujeres conocen más de hierbas no-boscosas, en especial, medicinales (Phillips \& Gentry, 1993).

Por rangos de edad, se encontró que las personas entre 19 y 54 años (rango I) conocen 25 especies de uso medicinal (75,8\%), una menor cantidad que las personas entre 55 y 77 años (rango 2), que conocen 31 especies (93,9\%). La prueba de $x^{2}$ muestra que no existen diferencias significativas en el conocimiento entre los dos rangos de edad en el número de plantas de uso medicinales conocidas $\left(X^{2} \mathrm{cal}\right.$ $=2,06 ; X^{2}$ th $\left.=3,84 ; g l=1 ; p=0,151\right)$. A pesar de esto, es importante la recopilación del saber popular y el rescate del uso tradicional de plantas medicinales que poseen los abuelos y que es transmitida de generación en generación. Resultados similares fueron reportados por Arango (2004) y por Angulo et al. (2012).

El estudio permite concluir que existe un importante conocimiento sobre el uso de plantas medicinales en las comunidades campesinas del área rural de la Comunidad San Jacinto del cantón Ventanas, provincia de Los Ríos, Ecuador. El cálculo del valor de uso (IVU), el índice de conocimiento relativo de las especies por varios informantes (RVU) y el nivel de uso significativo (UST) permitió identificar la importancia de 33 especies de plantas medicinales, reportadas para este estudio, siendo las especies más importantes la sábila, la hoja del aire, la ruda de gallinazo, el toronjil, la valeriana, la hierba luisa y el orégano (Tabla 2), especies que la comunidad utiliza para el diario vivir y, de las cuales, mantienen la tradición de su cultivo en huertos caseros. La lejanía de centros de salud de calidad, así como la reducida capacidad económica de los habitantes de la comunidad San Jacinto, hace que el uso de plantas medicinales para el tratamiento de afecciones y enfermedades sea una alternativa al uso de medicamentos modernos.

Tabla 2. Análisis cuantitativo de las más importantes plantas medicinales utilizadas por los habitantes del área rural de la Comunidad San Jacinto, cantón Ventanas, provincia de Los Ríos, Ecuador.

\begin{tabular}{|c|c|c|c|c|c|}
\hline Nombre científico & Planta & Citaciones & IVU & RVU & UST (\%) \\
\hline Aloe vera & Sábila & 24 & 1,60 & 0,45 & 52,17 \\
\hline Kalanchoe pinnata & Hoja del aire & 23 & 1,77 & 0,39 & 50,00 \\
\hline Ruta graveolens & $\begin{array}{l}\text { Ruda de } \\
\text { gallinazo }\end{array}$ & 20 & 2,00 & 0,30 & 43,48 \\
\hline Melissa officinalis & Toronjil & 15 & 1,00 & 0,45 & 32,61 \\
\hline Valeriana officinalis & Valeriana & 13 & 1,86 & 0,21 & 28,26 \\
\hline Cymbopogon citratus & Hierba luisa & 13 & 1,00 & 0,39 & 28,26 \\
\hline Origanum vulgare & Orégano & 12 & 1,00 & 0,36 & 26,09 \\
\hline Ruta chalepensis & $\begin{array}{l}\text { Ruda de } \\
\text { castilla }\end{array}$ & 12 & 2,00 & 0,18 & 26,09 \\
\hline Alternanthera cf. pubiflora & $\begin{array}{l}\text { Escancel } \\
\text { morado }\end{array}$ & 11 & 1,10 & 0,30 & 23,91 \\
\hline Dysphania ambrosioides & Paico & 10 & 1,11 & 0,27 & 21,74 \\
\hline
\end{tabular}

IVU: Índice de valor de uso. (RVU): conocimiento relativo de la especie por varios informantes. UST (\%): el nivel de uso significativo Tramil. 
Agradecimientos: Los autores agradecen de manera especial a las personas de la comunidad de San Jacinto, por el aporte de su conocimiento tradicional sobre las plantas medicinales de la zona. Conflictos de intereses: El manuscrito fue preparado y revisado con la participación de todos los autores, quienes declaramos que no existe ningún conflicto de intereses que ponga en riesgo la validez de los resultados presentados. Financiación: Este estudio fue realizado dentro del proyecto "Formas de uso y aprovechamiento de la biodiversidad en las provincias de Los Ríos y Santo Domingo de los Tsáchilas, Ecuador", apoyado por el Proyecto Prometeo de la Secretaría de Educación Superior, Ciencia, Tecnología e Innovación de la República del Ecuador.

\section{BIBLIOGRAFÍA}

1. AKARRETA, S.; CAVERO, R.; CALVO, M. 2007. First comprehensive contribution to medical ethnobotany of Western Pyrenees. J. Ethnobiol. Ethnomed. 3:26 DOI:10.1186/1746-4269-3-26.

2. ALMEIDA, E. 2000. Culturas prehispánicas del Ecuador. Viajes Chasquiguiñán Cía. Ltda., Quito. 180p.

3. ANGUlO, A.; ROSERO, R.; GONZÁleZ, M. 2012. Estudio etnobotánico de las plantas medicinales utilizadas por los habitantes del corregimiento de Genoy, Municipio de Pasto, Colombia. Rev. Universidad y Salud. 14(2):168-185.

4. ARANGO, S. 2004. Estudios etnobotánicos en los Andes Centrales (Colombia): Distribución del conocimiento del uso de las plantas según características de los informantes. Lyonia. 7(2):89-104.

5. ARMIJOS, CH.; COTA, L.; GONZÁLEZ, S. 2014. Traditional medicine applied by the Saraguro yachakkuna: a preliminary approach to the use of sacred and psychoactive plant species in the southern region of Ecuador. J. Ethnobiol. Ethnomed. 10:26 DOI:10.1186/1746-4269-10-26.

6. BÉJAR, E.; BUSSMANN, R.; ROA, C.; SHARON, D. 2002. Medicinal Herbs of Southern Ecuador. Latino Herbal Press, Spring Valley, CA. 352p.

7. BELLON, M.R. 1996. The dynamics of crop infraspecific diversity: A conceptual framework at the farmer level. Econ. Bot. 50(1):26-39.

8. BERNAL, H.Y.; GARCÍA, M.H.; QUEVEDO, S.F. 2011. Pautas para el conocimiento, conservación y uso sostenible de las plantas medicinales nativas en
Colombia: Estrategia nacional para la conservación de plantas. Instituto de Investigación de Recursos Biológicos Alexander von Humboldt. Bogotá, D. C., Colombia. 230p.

9. BEYRA, A.; LEÓN, M.; IGLESIAS, E.; FERRANDIZ, D.; HERRERA, R.; VOLPATO, D.; GODÍNEZ, D.; GUIMARAIS, S.; ÁLVAREZ, R. 2002. Estudios etnobotánicos sobre plantas medicinales en la provincia de Camagüey (Cuba). Anales Jardín Botánico Madrid. 61:185-203.

10. BHATTARAI, S.; CHAUDHARY, R.; QUAVE, C.; TAYLOR, R. 2010. The use of medicinal plants in the transhimalayan arid zone of Mustang district, Nepal. J. Ethnobiol. Ethnomed. 6:14 doi:10.1186/17464269-6-14.

11. BUSSMANN, R.; SHARON, D. 2006. Traditional medicinal plant use in Loja province, Southern Ecuador. J. Ethnobiol. Ethnomed. 2:47 doi:10.1186/17464269-2-47.

12. CENTRAL ECUATORIANA DE SERVICIOS AGRÍCOLA -CESA- (ed). 1992. Usos tradicionales de las especies forestales nativas en el Ecuador. Tomo 2. FEPP, Quito.

13. CERÓN MARTÍNEZ, C.E. 2006. Plantas medicinales de los Andes ecuatorianos. En: Moraes, M.; Øllgaard, B.; Kvist, L.P.; Borchsenius, F.; Balslev, H. (Editores), Botánica Económica de los Andes Centrales. Universidad Mayor de San Andrés, La Paz. p.285293.

14. CERÓN MARTÍNEZ, C.E. 2002. Etnobotánica de la comunidad de Alao, zona de influencia del Parque Nacional Sangay. Cinchonia. 3(2): 55-63.

15. CERÓN, C.; MONTALVO, C. 1998. Etnobotánica de los Huaorani de Quehueiri-ono Napo-Ecuador. AbyaYala, Quito. 232p.

16. DOMÍNGUEZ-VÁZQUEZ, G.; CASTRO-RAMÍREZ, A. 2002. Usos medicinales de la familia Labiatae en Chiapas, México. Etnobiología (2):19-31.

17. DE LA TORRE, L.; MURIEL, P.; BALSLEV, H. 2006. Etnobotánica en los Andes del Ecuador. En: Moraes, M.; Øllgaard, B.; Kvist, L.P.; Borchsenius, F.; Balslev, H. (Editores), Botánica Económica de los Andes Centrales. Universidad Mayor de San Andrés, La Paz. p.246-267. 
18. DE LA TORRE, L.; ALARCÓN, D.; PETER-KVIST, L.; SALAZAR LECARO, J. 2008. Usos medicinales de las plantas. En: de la Torre, L.; Navarrete, H.; Muriel, P.; Macía, M.J.; Balslev, H. (eds.). Enciclopedia de las Plantas Útiles del Ecuador. Herbario QCA7 \& Herbario AAU. Quito \& Aarhus. p.105-114.

19. ESTRELLA, E. 1991. Flora huayaquilensis. La expedición botánica de Juan Tafalla a la Real Audiencia de Quito 1799-1808. Abya-Yala, Centro Cultural Artes, Quito. 103p.

20. GALEANO, G. 2000. Forest use at the Pacific Coast of Chocó, Colombia: a Quantitative Approach. Econ. Bot. 54(3):358-376.

21. GENTRY, A.H. 1982. Neotropical floristic diversity: Phytogeographical connections between Central and South America, pleistocene climatic fluctuations, or an accident of the Andean orogeny. Ann. Missouri Bot. Garden. (69):557-593.

22. GERMOSÉN-ROBINEAU, L. (Ed). 1995. Hacia una farmacopea vegetal caribeña. Edición TRAMIL 7. Enda - Caribe, UAG \& Universidad de Antioquia. Santo Domingo. 696p.

23. HAMMER, Ø.; HARPER, D.A.T.; RYAN, P.D. 2001. PAST: Paleontological Statistics Software Package for Education and Data Analysis. [Computer program] Palaeontología Electrónica. Disponible desde Internet en: http://palaeoelectronica.org/2001_1/past/ issue1_01.htm (con acceso 23/05/2014).

24. HERNÁNDEZ, J.; VOLPATO, G. 2004. Herbal mixtures in the traditional medicine of Eastern Cuba. J. Ethnopharmacol. 90:293-316.

25. HOLDRIDGE, L. 1987. Ecología basada en las zonas de vida. San José, Costa Rica, IICA. 216p.

26. HURTADO-ULLOA, R.; MORAES, R.M. 2010. Comparación del uso de plantas por dos comunidades campesinas del bosque tucumano - boliviano de Vallegrande (Santa Cruz, Bolivia). Ecología en Bolivia $45(1): 20-54$.

27. INSTITUTO NACIONAL DE ESTADÍSTICAS Y CENSOS -INEC-. 2010. Censo de Población y Vivienda 2010.

28. JORGENSEN, P.; LEÓN-YÁNEZ, S. 1999. Catalogue of the Vascular Plants of Ecuador. Missouri Botanical Garden Press, U.S.A, 1181p.
29. LAGOS-LÓPEZ, M. 2007. Estudio etnobotánico de especies vegetales con propiedades medicinales en seis municipios de Boyacá, Colombia. Actualidades Biol. 29(86):87-96.

30. LULEKAL, E.; KELBESSA, E.; BEKELE, T.; YINEGER, H. 2008. An ethnobotanical study of medicinal plants in Mana Angetu District, southeastern Ethiopia. J. Ethnobiol. Ethnomed. 4:10 DOI: 10.1186/1746-4269-4-10.

31. MADSEN, J.E.; MIX, R.L.; BALSLEV, H. 2001. Flora of Puna Island. Plant Resources on a Neotropical Island. Aarhus University Press, Aarhus. 304p.

32. MARÍN-CORBA, C.; CÁRDENAS-LÓPEZ, D.; SUÁREZ-SUÁREZ, S. 2005. Utilidad del valor de uso en etnobotánica. Estudio en el Departamento de Putumayo (Colombia). Caldasia. 27(1):89-101.

33. MONCAYO, N.; ZAMBRANO, J.; PATIÑO, A. 2006. Plantas medicinales empleadas por los campesinos de los corregimientos de Casabuy, Hato Viejo y Sánchez del municipio de Chachagüí. Rev. Centro de Estudios en Salud 1(7):15-33.

34. NARANJO, P.; CRESPO, A. 1997. Etnomedicina, Progresos Italo-Latinoamericanos, vol. I. Abya Yala, Quito. 257p.

35. NARANJO, P.; ESCALERAS, R. 1995. La Medicina Tradicional en el Ecuador. Corporación Editora Nacional, Quito. 192p.

36. PHILLIPS, O. 1996. Some quantitative methods for analysing ethnobotanical knowledge. In: Alexiades, M.N. (ed.) Selected guidelines for ethnobotanical research: A field manual. The New York Botanical Garden, Bronx, New York. p.171-197.

37. PHILLIPS, O.; GENTRY, A.H. 1993. The useful plants of Tambopata, Peru: I. Statistical hypothesis tested with a new quantitative technique. Econ. Botany. 47(1):15-32.

38. PINEDO-VÁSQUEZ, M.; ZARIN, D.; JIPP, P.; CHOTAINUMA, J. 1990. Use-values of tree species in a comunal forest reserve in northeast Peru. Conserv. Biology. 4(4):405-415.

39. RÍOS, M.; DE LA CRUZ, R.; MORA, A. 2008. Conocimiento Tradicional y Plantas útiles del Ecuador: Saberes y Prácticas. IEPI y Ediciones Abya - YalaQuito, Ecuador. 79p. 
40. RIVERA, N.D.; OBÓN DE, C.C. 1991. La guía de INCAFO de las plantas útiles y venenosas de la Península Ibérica y Baleares. INCAFO Archivo Fotográfico. Madrid, España. 1257p.

41. SALDANHA, L.; FARIAS, R.; DE ALBUQUERQUER, U.P. 2005. Knowledge and use of medicinal plants by local specialists in a region of Atlantic Forest in the state of Pernambuco (Northeastern Brazil). J. Ethnobiol Ethnomed. 1:9 DOI: 10.1186/17464269-1-9.

42. SANTÍN, F.M. 2004. Ethnobotany of the communities of the upper Rio Nangaritza. Lyonia. 7(2):105-125.

43. STEVENS, P.F. 2001-2013. Angiosperm Phylogeny Website. Version 13. Disponible desde Internet en: http://www.mobot.org/MOBOT/research/APweb/ (con acceso 05/05/2013).

44. SUÁREZ-DUQUE, D. 2008. Diferencias en el uso de plantas entre hombres y mujeres en una comunidad de pie de monte del norte del Ecuador. J. Botanical Res. Inst. Texas. 2(2):1295-1308.
45. TENE, V.; MALAGÓN, O.; VITA-FINZI, P.V.; VIDARI, G.; ARMIJOS, CH.; ZARAGOZA, T. 2007. An ethnobotanical survey of medicinal plants used in Loja and Zamora-Chinchipe, Ecuador. J. Ethnopharmacol. 111:63-81.

46. TOSCANO-GONZÁLEZ, J. 2006. Uso tradicional de plantas medicinales en la vereda San Isidro, municipio de San José de Pare-Boyacá: un estudio preliminar usando técnicas cuantitativas. Acta Biológica Col. 11(2):137-146.

47. VALDÉS-COBOS, A. 2013. Conservación y uso de plantas medicinales: el caso de la región de la Mixteca Alta Oaxaqueña, México. Ambiente y Desarrollo, Bogotá (Colombia). 17(33):87-97.

48. VILLACRÉS, V. (Coordinator). 1995. Bioactividad de plantas amazónicas. Abya Yala, Quito. 378p.

Recibido: Diciembre 18 de 2014

Aceptado: Abril 28 de 2015

Cómo citar:

Paredes, D.J.; Buenaño-Allauca, M.P.; Mancera-Rodríguez, N.J. 2015. Usos de plantas medicinales en la comunidad San Jacinto del Cantón Ventanas, Los Ríos - Ecuador. Rev. U.D.C.A Act. \& Div. Cient. 18(1): 39-50. 